\section{The New Maneuver for Difficult Intubation}

\section{Abstract}

It is believed that the airway management is more difficult in obese than nonobese patients due to the anatomic changes resulting from overweight. In this investigation was studied the new maneuver for difficult tracheal intubation in one obese patient. An 85 year old obese and diabetic man with cardiopulmonary arrest and without history of trauma was brought to the emergency department (ED). In this patient, the neck circumference was greater and sternomental distance was shorter than ordinary individuals. The cardiopulmonary resuscitation (CPR) was begun for patient immediately. In this patient, tracheal intubation with the conventional method was unsuccessful. Then, the new method (swan neck maneuver) for difficult intubation was attempted in patient successfully. In this maneuver, the upper part of the back of patient was elevated with bolsters so that the neck of patient was extended at ease and pharyngeal axis was perch along with laryngeal axis. Then, intubation was performed in this position simply. It looks that this new maneuver can perform easily for difficult intubations in obese patients without history of trauma.

Keywords: Difficult intubation; Swan neck maneuver; Obesity

\section{Seyed Farshad Heidari}

\author{
Mazandaran University of Medical Sciences, \\ Sari, Mazandaran, Iran
}

Corresponding author:

Seyed Farshad Heidari

” s.f.heidari@gmail.com

Mazandaran University of Medical Sciences, Sari, Mazandaran, Iran.

Tel: 988338224670

Fax: 9188317379

Citation: Heidari SF. The New Maneuver for Difficult Intubation. J Intensive \& Crit Care 2016, 2:3.

\section{Introduction}

It is believed that the airway management is more laborious in obese than other patients due to the anatomic changes resulting from high weight [1]. In obese patients, there is an inverted relevance between weight and pharyngeal area due to fat deposition on cervical structures [2]. However, large neck circumference and modified Mallampati index (grade III or IV) are predictors that established as risk factors for difficult laryngoscopy $[1,3]$. Anatomical measurements, such as reduced mouth opening, shorter sternomental, and thyromental distances and greater neck circumference are highly related to diagnosis of difficult airway access [2]. In this disquisition was studied the new maneuver for difficult tracheal intubation in one obese patient that intubation in the usual approach failed.

\section{Case Report}

An 85 year-old diabetic and obese man with cardiopulmonary arrest and without history of trauma was brought to the emergency department (ED). The patient was transferred to the cardiopulmonary resuscitation (CPR) room and CPR was begun for patient immediately. Chest compression and bagmask ventilation was begun for patient instantly. Then cardiac monitoring and pulse oximetry was performed for patient. In this patient, the neck circumference was greater and sternomental distance was shorter than ordinary individuals and did not able to the extension of the neck. Therefore, tracheal intubation with traditional method was unsuccessful in this patient [4]. Then, the new method (swan neck maneuver) was tried for intubation in this patient that bolsters were placed under the upper part of the back of patient immediately so that the chest found a ramp (angle 30 deg approximately) with horizon level. The neck of patient was extended at ease and pharyngeal axis was perch along with laryngeal axis. Then, anterior-superior upward force was inducted in this position with the help of direct laryngoscopy that the vocal cords were seen and endotracheal intubation by tracheal tube with internal diameter $8 \mathrm{~mm}$ was performed in this position simply.

\section{Discussion}

In this case report was perused the new maneuver for difficult tracheal intubation in one obese and diabetic patient that intubation in the usual approach failed. In this new method (swan neck maneuver), cushions places under the upper part of the back of patient hastefully so that the chest find an inclined plane (angle 30 degree, approximately) with horizon level. The neck of patient extend easily and pharyngeal axis places along with laryngeal axis. Afterwards, anterior-superior upward force inducts in this position with the help of direct laryngoscopy that the vocal cords behold and tracheal intubation perform 
in this position simply that all of this proceedings last $30 \mathrm{sec}$. Resuscitation activities include chest compression continued during this period. Despite the plentiful efforts taken for patient, unfortunately, a resuscitation proceeding was not successful and patient was expired. Difficult intubation, sometimes defined as inadequate glottis exposure to direct laryngoscopy, is more prevalent in patients with high BMI [1]. Theories to explain difficult intubation in obese patients include: fatty tissue in the neck, chest, abdomen, and pharynx may compromise intubation [5]; limited neck extension, anatomic abnormality, and tissue reactivity [6]; fat distribution [7]; large necks [8]; diabetes mellitus [9]. These factors are believed to impress laryngoscopy and therefore tracheal intubation [3]. The anatomical parameters of airway evaluation showed no difference between obese and non-obese patients. The only exception was the value of neck circumference and Mallampati index, which were higher in the obese patients [10]. This may be explained by the reality that these patients had decreased pharyngeal area due to the augmentation of soft tissue in this region [2]. The patients who had the following three important factors influenced the possibility of a difficult intubation: neck circumflex greater than $40 \mathrm{~cm}$, diabetes mellitus, and high overweight [9].

Accordingly, swan neck maneuver was applied successfully for difficult intubation in one diabetic patient with high overweight that intubation in the traditional approach was failed. It seems that this new maneuver can perform at ease for difficult tracheal intubations in obese patients without history of trauma. 


\section{References}

1 Brodsky JB, Lemmens HJ, Brock-Utne JG (2002) Morbid obesity and tracheal intubation. Anesth Analg 94: 732-736.

2 Benumof JL (2001) Obstructive sleep apnea in the adult obese patient: Implications for airway management. Clin Anesth 13: 144-156.

3 Juvin P, Lavaut E, Dupont $\mathrm{H}$ (2003) Difficult tracheal intubation is more common in obese than in lean patients. Anesth Analg 97: 595-600.

4 Collins JS, Lemmens HJ, Brodsky JB (2004) Laryngoscopy and morbid obesity: A comparison of the "sniff" and "ramped" positions. Obes Surg 14: 1171-1175.

5 Kristensen MS (2010) Airway management and morbid obesity. Eur J Anaesthesiol 27: 923-927.

6 Hekiert AM, Mandel J, Mirza N (2007) Laryngoscopies in the obese:
Predicting problems and optimizing visualization. Ann Otol Rhinol Laryngol 116: 312-316.

7 Ezri T, Medalion B, Weisenberg M (2003) Increased body mass index per se is not a predictor of difficult laryngoscopy. Can J Anaesth 50: 179-183.

8 Gonzales H, Minville V, Delanoue K (2008) The importance of neck circumference to intubation difficulties in obese patients. Anesth Analg 106: 1132-1136.

9 Mashour GA, Kheterpal S, Vanaharam V (2008) The extended Mallampati score and a diagnosis of diabetes mellitus are predictors of a difficult laryngoscopy in the morbidly obese. Anesth Analg 107: 1919-1923.

10 Kim WH, Ahn HJ, Lee CJ (2011) Neck circumference to thyromental distance ratio: A new predictor of difficult intubation in obese patients. Br J Anaesth 106: 743-748. 